\title{
(2) OPEN ACCESS \\ Multisite medical record review of emergency department visits for unspecified injury of head following the ICD-10-CM coding transition
}

\author{
Alexis Peterson 다, ${ }^{1}$ Barbara A Gabella 다, ${ }^{2}$ Jewell Johnson, ${ }^{2}$ Beth Hume, ${ }^{3}$ Ann Liu, \\ Julia F Costich, ${ }^{5}$ Jeanne Hathaway, ${ }^{6}$ Svetla Slavova $\left[10,{ }^{7}\right.$ Renee Johnson, ${ }^{1}$ \\ Matt Breiding
}

${ }^{1}$ National Center for Injury Prevention and Control-Division of Injury Prevention, Centers for Disease Control and Prevention, Atlanta, Georgia, USA ${ }^{2}$ Colorado Department of Public Health and Environment, Denver, Colorado, USA ${ }^{3}$ Massachusetts Department of Public Health, Boston, Massachusetts, USA

${ }^{4}$ Center for Environmental \& Occupational Epidemiology, Environmental Health Bureau, Maryland Department of Health, Baltimore, Maryland, USA ${ }^{5}$ Kentucky Injury Prevention and Research Center, University of Kentucky, Lexington, Kentucky, USA

${ }^{6}$ Injury Surveillance Program, Massachusetts Department of Public Health, Boston Massachusetts, USA

University of Kentucky Lexington, Kentucky, USA

Correspondence to Dr Alexis Peterson, National Center for Injury Prevention and Control-Division of Injury Prevention, Centers for Disease Control and Prevention, Atlanta, GA 30329-4018,USA; yxf5@ cdc.gov

Received 24 February 2020 Revised 4 August 2020 Accepted 7 August 2020

\begin{abstract}
Introduction In 2016, a proposed International Classification of Diseases, Tenth Edition, Clinical

Modification surveillance definition for traumatic brain injury (TBI) morbidity was introduced that excluded the unspecified injury of head (S09.90) diagnosis code. This study assessed emergency department (ED) medical records containing S09.90 for evidence of TBI based on medical documentation.
\end{abstract}

Methods State health department representatives in Maryland, Kentucky, Colorado and Massachusetts reviewed a target of 385 randomly sampled ED records uniquely assigned the S09.90 diagnosis code (without proposed TBI codes), which were initial medical encounters among state residents discharged home during October 2015-December 2018. Using standardised abstraction procedures, reviewers recorded signs and symptoms of $\mathrm{TBI}$, and head imaging results. A tiered case confirmation strategy was applied that assigned a level of certainty (high, medium, low, none) to each record based on the number and type of symptoms and imaging results present in the record. Positive predictive value (PPV) of S09.90 by level of TBI certainty was calculated by state.

Results Wide variation in PPV of sampled ED records assigned S09.90: $36 \%-52 \%$ had medium or high evidence of $\mathrm{TBI}$, while $48 \%-64 \%$ contained low or no evidence of a TBI. Loss of consciousness was mentioned in $8 \%-24 \%$ of sampled medical records.

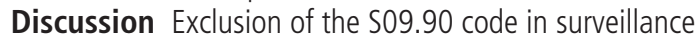
estimates may result in many missed TBI cases; inclusion may result in counting many false positives. Further, missed TBI cases influenced by incidence estimates, based on the TBI surveillance definition, may lead to inadequate allocation of public health resources.

\section{INTRODUCTION}

Traumatic brain injury (TBI), a disruption of the normal function of the brain due to an external force, can be temporary or have life-long consequences. ${ }^{1}$ TBI is preventable (https://www.cdc.gov/ traumaticbraininjury/prevention.html). State and federal public health agencies monitor the burden of TBI and identify risk factors in a population, using public health surveillance definitions and existing data sources, such as administrative billing claims for emergency department (ED) visits and hospitalisations. On 1 October 2015 in the USA, morbidity diagnosis coding in billing claims transitioned to the International Classification of Disease, Tenth Revision, Clinical Modification (ICD-10-CM). ${ }^{2}$ This necessitated the development of a new surveillance definition for TBI morbidity.

In 2016, the CDC proposed an ICD-10-CM surveillance definition for TBI morbidity that excludes the diagnosis code for 'unspecified injury of head' (S09.90), ${ }^{3}$ although the previous ICD-9-CM TBI morbidity surveillance definition included its complementary ICD-9-CM diagnosis code (959.01). ${ }^{1}$ In the ICD-9-CM coding era, multiple studies found that the 'unspecified injury to the head' codes represented a majority $(50 \%-58 \%)$ of TBI morbidity records identified in their samples. ${ }^{4-6}$ Notably, in a prospective cohort study, only $25 \%$ of cases assigned the diagnosis code 'unspecified injury to the head' (ICD-9-CM 959.01 code) met the clinical definition of $\mathrm{TBI}^{5}$ Currently, the positive predictive value (PPV) of the unspecified injury of head ICD-10-CM S09.90 diagnosis code for identifying TBI morbidity is unknown. Given concerns about the validity of this code in ICD-9-CM, the purpose of this study was to assess a sample of ED medical records, identified from administrative billing data and assigned diagnosis code S09.90 (without the proposed TBI codes (referred to as uniquely assigned throughout the manuscript text and tables)), for evidence of TBI based on medical documentation in four states. At the national surveillance level, the effect of excluding the unspecified injury of head S09.90 code from the CDC's TBI morbidity surveillance case definition, on the magnitude of TBI-related ED visits, is unknown. Understanding the PPV of the $\mathrm{S} 09.90$ code for TBI in the ED is of paramount importance as prevention strategies and resources are influenced by incidence estimates based on the TBI surveillance case definition.

\section{METHODS}

State health department representatives in Maryland, Kentucky, Colorado and Massachusetts conducted a review of randomly sampled medical records to calculate the PPV of cases assigned code S09.90 (unspecified injury of head) without the proposed TBI codes. In coordination with CDC, the four states developed a study protocol that included sample selection criteria, a tiered TBI case confirmation strategy, an abstraction form and reviewer 
instructions to standardise the process across states. Patients or the public were not involved in the design, or conduct, or reporting, or dissemination plans of our research.

\section{Study protocol}

Sample selection

Sample selection criteria included ED patients with an ICD-10-CM diagnosis code uniquely assigned S09.90 in any diagnosis field, an initial medical encounter (ie, a seventh character of 'A' or missing seventh character) and who were state residents and discharged home during October 2015-December 2018. A random sample target of 385 records per state was chosen to provide sufficient records to achieve a 95\% CI with a $3 \%$ precision around the PPV estimate. Stratification was considered unnecessary for the review.

Medical record review processes varied in each state. To conduct this review, Maryland reviewers received access to patient electronic health records (EHRs) in the Epic system. Kentucky used full EHRs from a two-hospital system that included a level 1 trauma centre. Colorado reviewers accessed full EHRs from some hospitals, and for other hospitals, Colorado requested the full ED report, ambulance/emergency medical technician (EMT) record (Emergency medical services (EMS) transport record), face sheet, radiology reports and toxicology reports. Massachusetts requested medical documents like those requested by Colorado from acute care hospitals in the state.

\section{Abstraction form}

The states developed an abstraction form to record all medical record review documentation (see online supplementary appendix 1). Reviewed records included a confirmed recent injury event. Records with insufficient documentation of an injury event were not reviewed. Fourteen TBI-related signs and symptoms, positive TBI imaging results from a CT scan, MRI, or X-ray, and whether a TBI assessment tool was used in the ED were recorded.

Medical record reviewers from each state used a detailed reviewer manual (the initial detailed draft of a manual for the medical record review of TBI-related diagnoses was based on the multisite common abstraction form used in this study and a related Colorado study of the CDC proposed TBI ICD-10-CM case definition; see online supplementary appendix 2) drafted by Colorado that was finalised with the assistance of the other three states and the CDC TBI team. The manual described each variable to be collected and was used across the four states to standardise results. Based on input from subject matter experts, a TBI Imaging Definition was developed that included a list of clinical terms and phrases to assist medical record reviewers in assessment of results from a CT, MRI or X-ray image as positive or negative for TBI.

\section{TBI case confirmation strategy}

A tiered TBI case confirmation strategy was developed to assign a level of certainty to each record based on the number and type of symptoms and CT/MRI/X-ray imaging present in the record (table 1). This strategy aimed to represent the amount/quality of medical evidence documented in the record and the inherent uncertainty in making a TBI diagnosis at the milder end of the injury severity spectrum. Representing this uncertainty may be particularly important for diagnoses such as unspecified injury of head which can be used by physicians when they are uncertain as to whether a mild TBI has occurred. Signs and symptoms were separated into two groups. Group 1 included those symptoms more definitive of a TBI: loss of consciousness (LOC); being dazed, foggy or confused; or having memory problems. ${ }^{7}$ Group 2 signs and symptoms are less definitive in determining whether a TBI occurred. These included nausea/vomiting, headache or pressure in head, dizziness/poor balance, change in vision, poor concentration, sensitivity to noise or light, irritability or change in mood or personality, drowsiness or change in sleep, speech problems, hearing problems or weakness/numbness.

\section{Analysis}

The four states, along with the CDC TBI Team, developed an analysis plan and a corresponding template for states to review results, including counts and percentages. To answer the central question, what percentage of reviewed records assigned an ICD-10-CM code for unspecified head injury (S09.90XA, without the proposed TBI codes) in the ED contain documented evidence of a TBI, the percentage of cases by level of TBI

Table 1 Groups 1 and 2 traumatic brain injury (TBI)-related signs and symptoms and criteria used for categorisation of levels of certainty of TBI in sampled emergency department and shock trauma centre medical records uniquely assigned International Classification of Disease, Tenth Revision, Clinical Modification code S09.90 ('unspecified injury of head')

\begin{tabular}{|c|c|c|c|c|}
\hline \multicolumn{5}{|c|}{ Groups 1 and 2 TBI-related signs and symptoms } \\
\hline $\begin{array}{l}\text { Group } 1 \text { signs/ } \\
\text { symptoms }\end{array}$ & $\begin{array}{l}\text { Dazed/foggy/confused } \\
\text { Memory problems } \\
\text { Loss of consciousness }\end{array}$ & & & \\
\hline \multicolumn{5}{|c|}{ Criteria and categorisation of levels of certainty of TBI: } \\
\hline & Documentation of injury event? & Group 1 signs/symptoms & Group 2 signs/symptoms & $\mathrm{CT}$, MRI or X-ray imaging is positive for TBI? \\
\hline High & Yes (and) & 1 or more (or) & 3 or more (or) & Yes \\
\hline Medium & Yes (and) & N/A & 2 (or) & Suspected \\
\hline $\begin{array}{l}\text { Low } \\
\text { None }\end{array}$ & Yes (and) & $N / A$ & Only 1 (and) & No \\
\hline
\end{tabular}

CT scan, Computed tomography; MRI, Magnetic resonance imaging; N/A, not applicable. 
Table 2 State-based details for medical record review of charts uniquely assigned 'unspecified injury of head' International Classification of Disease, Tenth Revision, Clinical Modification S09.90 code

\begin{tabular}{|c|c|c|c|c|}
\hline Characteristic & Maryland & Kentucky & Colorado & Massachusetts \\
\hline Dates & $\begin{array}{l}\text { January 2016-December } \\
2018\end{array}$ & January 2016-June 2018 & $\begin{array}{l}\text { January 2017-December } \\
2017\end{array}$ & $\begin{array}{l}\text { October 2015-September } \\
2016\end{array}$ \\
\hline Type of hospital & Large teaching hospital* & Tertiary care and community hospital & All acute care hospitals & All acute care hospitals \\
\hline Number of sampled hospitals & 2 & 2 & 54 & 73 \\
\hline$\%$ of state population covered by sampled hospitals & $14.5 \% \dagger$ & $38 \%$ & $100 \%$ & $100 \%$ \\
\hline Number of diagnosis fields & 30 & 25 & 30 & 34 \\
\hline $\begin{array}{l}\text { Medical record } \\
\text { reviewer }\end{array}$ & 18 clinical researchers & 2 trauma nurses & 1 professional coder & 3 professional coders \\
\hline
\end{tabular}

*Maryland reviewed records from emergency department visits and a shock trauma centre.

tMaryland calculated the percentage of patient records corresponding to the two sampled hospitals out of the total records in a year within their healthcare administrative database.

certainty was calculated. Percentages for case counts less than 11 were suppressed. SAS V.9.3 or V.9.4, and IBM SPSS V.23.0 were used for data analysis.

\section{Variations in methodology across states}

Data access, data quality or other considerations impacted an individual state's ability to fully standardise to the study protocol. Table 2 provides an overview of state variation in study methodology.

\section{Maryland}

Maryland collaborated with the University of Maryland School of Medicine National Study Center for Trauma and Emergency Medical Systems (UMNSC) and the Johns Hopkins Center for Injury Research and Policy (JHCIRP) to conduct chart reviews at the University of Maryland Medical System and Johns Hopkins Hospital, respectively. Ten medical records reviewers from UMNSC and eight from JHCIRP were involved in data abstraction. Two lead abstractors at UMNSC trained the JHCIRP reviewers and coordinated the medical records review process at both institutions.

The unique circumstances of the collaboration between Maryland Department of Health, UMNSC and JHCIRP required deviations from the overall study protocol. At the University of Maryland Medical System, TBI cases were obtained from the R Adams Cowley Shock Trauma Center (STC). STC is the primary adult neurotrauma referral centre for the state of Maryland, and state EMS triage and transfer protocols send the majority of applicable TBI study cases directly to STC. Data from Johns Hopkins Hospital ED, the state's referral centre for paediatric trauma, were used according to the study protocol.

UMNSC identified 56 cases assigned a diagnosis code of S09.90 and all were reviewed, as well as a random sample of 296 cases from JHCIRP. At UMNSC, data from 2016 through 2018 were included in the study, while at JHCIRP data from 2016 through 2017 were included.

\section{Kentucky}

Kentucky's review included records from a two-hospital system with a level 1 trauma centre that provides care for most brain injury cases in the eastern half of the state. Review of sampled records were conducted by two experienced trauma nurses on staff at the participating hospitals. In compliance with Kentucky law, the use of participating hospital staff was necessary as public health authorities are not granted full medical record access in the absence of a public health emergency or court order.

\section{Colorado}

Colorado had several records that were coded as an initial encounter but on review, were found to be either subsequent encounters or the patient was not discharged home. These records were excluded from all analyses.

\section{Massachusetts}

Massachusetts was unable to distinguish initial medical encounters (denoted by a seventh character in the diagnosis code) except for those in the first-listed diagnosis field. All additional diagnosis fields were truncated to six alphanumeric characters.

\section{RESULTS}

Approximately, 80\% (Maryland's paediatric cases came from Johns Hopkins Hospital only), 52\%, 30\% and 24\% of sampled records from Maryland, Kentucky, Colorado and Massachusetts, respectively, were among paediatric ( $<16$ years) patients. Documentation of 'high certainty' evidence indicative of a TBI in charts assigned diagnosis code S09.90 varied among states. Approximately $32 \%, 22 \%, 36 \%$ and $16 \%$ of medical records from Maryland, Kentucky, Colorado and Massachusetts, respectively, had any group 1 sign/symptom (table 3). LOC was the most prevalent group 1 sign/symptom, documented in $8 \%-24 \%$ of medical records across the four states. Of all reviewed records, approximately 63\%, 52\%, 59\% and 71\% from Maryland, Kentucky, Colorado and Massachusetts had any group 2 sign/ symptom, respectively. Among the group 2 signs/symptoms, the most prevalent sign/symptom documented across all four states was headache/pressure in the head, ranging from nearly $35 \%$ to $61 \%$ of reviewed medical records. Nearly $65 \%$ of medical records reviewed in Maryland did not have evidence of imaging and $33 \%$ of total records had evidence of imaging but had no indication of TBI. Nearly 59\% of medical records reviewed in Kentucky did not have evidence of imaging and $41 \%$ of total records had evidence of imaging but had no indication of TBI. The vast majority $(97 \%)$ of medical records reviewed in Colorado did not contain evidence imaging was conducted. Nearly $41 \%$ of medical records reviewed in Massachusetts did not contain evidence of imaging and 59\% of the remaining records contained evidence of imaging but had no indication of TBI.

After applying the most conservative criteria to the medical records, approximately 37\%, 27\%, 41\% and 23\% of sampled records from Maryland, Kentucky, Colorado and Massachusetts, respectively, contained evidence of a TBI with high certainty (table 4). In addition, approximately 10\%, 10\%, $11 \%$ and $13 \%$ of sampled medical records from Maryland, 
Table 3 Frequency and proportion of signs/symptoms and imaging findings present within a sample of medical records uniquely assigned 'unspecified injury of head' International Classification of Disease, Tenth Revision, Clinical Modification S09.90 code, by state*

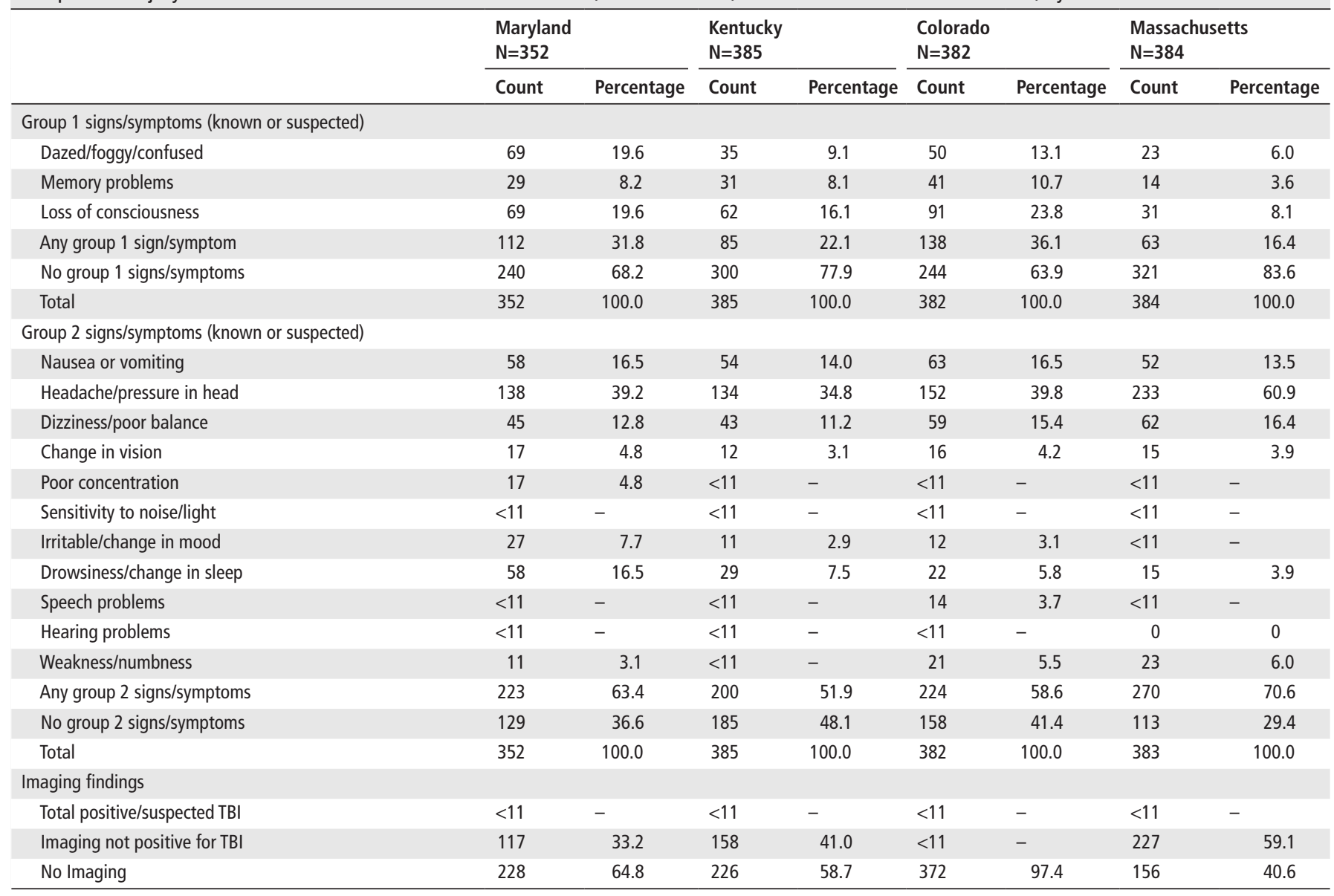

*Due to the extent of methodological differences between the states, authors are unable to account for the variation in results seen across states. For this reason, state-specific results should not be compared. Percentages for case counts less than 11 were suppressed.

$\mathrm{TBI}$, traumatic brain injury.

Kentucky, Colorado and Massachusetts, contained evidence of a TBI with 'medium certainty', respectively. Approximately 23\%, 23\%, 19\% and 38\% of medical records from Maryland, Kentucky, Colorado and Massachusetts had 'low certainty' evidence of a TBI, respectively. Results presented here should not be interpreted as TBI prevalence for each state as they were drawn among a sample of cases.

\section{DISCUSSION}

An ongoing challenge in accurately identifying non-fatal TBI cases in healthcare administrative data is the frequent use of the 'unspecified injury to the head' ICD diagnostic code. Multiple studies using the ICD-9-CM TBI morbidity surveillance case definition found that the 'unspecified' codes comprised a majority (50\%-58\%) of TBI cases in their sample. ${ }^{4-6}$ In 2016 , a proposed

Table 4 Positive predictive value of a traumatic brain injury (TBI), based on the high, medium and low level of certainty, in emergency departments and a shock trauma centre among a sample of medical records uniquely assigned International Classification of Disease, Tenth Revision, Clinical

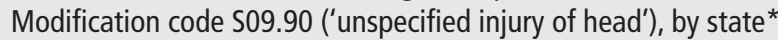

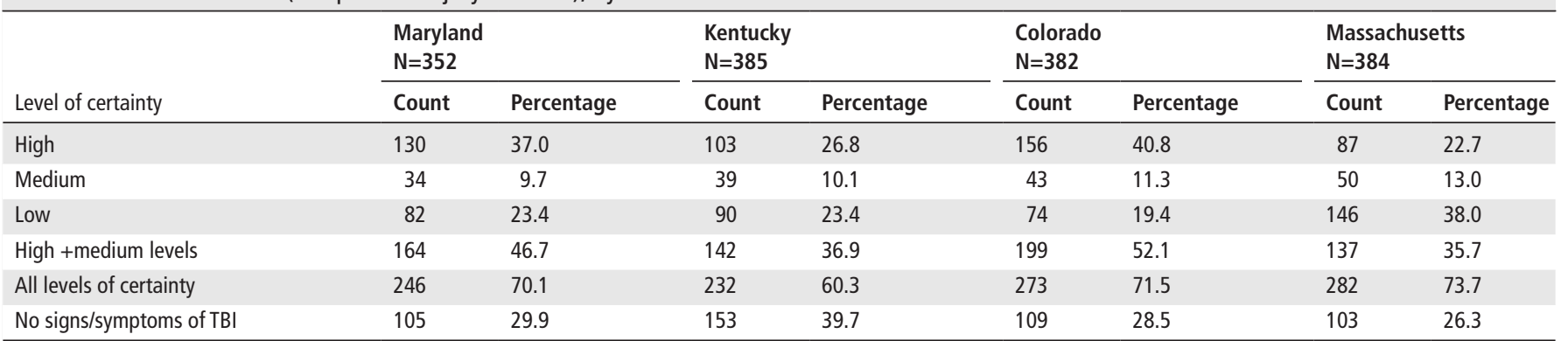

${ }^{*}$ Due to the extent of methodological differences between the states, authors are unable to account for the variation in results seen across states. For this reason, state-specific results should not be compared. 
ICD-10-CM-based surveillance definition for TBI morbidity was introduced that excluded the unspecified injury of head (S09.90) diagnosis code. ${ }^{3}$ To examine the use of this code subsequent to the switch to ICD-10-CM, the current study randomly sampled charts uniquely assigned the S09.90 code (unspecified injury of head) and found wide variation in PPV; among the four states, $23 \%-41 \%$ of sampled records contained high certainty evidence of TBI in the medical record. This finding is consistent with a previous prospective cohort study of patients presenting to an urban academic ED. ${ }^{5}$ Bazarian et al found that 75\% (299 of 397) of charts assigned ICD-9-CM code 959.01, 'head injury, unspecified', did not meet the clinical definition of mild TBI based on the absence of a documented LOC, mental status change or amnesia. $^{5}$

Combining sampled cases with 'high and medium certainty' evidence of TBI resulted in a PPV of approximately 36\%-52\% among the states. These results suggest that among the large number of head injuries, coded solely with S09.90, a substantial proportion of these records contain medium-to-high levels of documented evidence that a TBI occurred. In addition, there were a significant number of sampled charts assigned S09.90 for which there was low (ie, one TBI sign/symptom) or no documented evidence of TBI symptomatology. Results of this study present a significant conundrum for ICD-10-CM-based surveillance of TBI-related ED visits given: (1) the large number of unspecified injury of head diagnoses in the USA annually ${ }^{8}$ and (2) the need to include all or none of these cases in incidence estimates due to the inclusion/exclusion of this code in the surveillance case definition. Overall, the decision to include or exclude cases with S09.90, as the sole TBI-related code, will have profound effects on estimates of TBI-related ED visits. Inclusion of S09.90 in the surveillance definition would result in the inclusion of cases for which there is little to no evidence of a TBI in the record; exclusion of the code would result in the omission of cases for which there is moderate-to-strong evidence of TBI in the record.

An important limitation of this study was that determination of case inclusion was based solely on documentation included in the medical record. For multiple reasons, the sign/symptoms documented in a record may not reflect the totality of signs/symptoms experienced by a patient after a head injury. First, some patients are seen in the ED soon after an injury and some signs/symptoms do not manifest until hours or days after the injury. Second, the absence of symptom documentation in the medical record may indicate this symptom did not occur, was brief, or was significant but not recorded. Third, it has been shown that a significant proportion of true TBI cases in the ED are not assigned a TBI code. ${ }^{59}$ A 2018 ED-based study conducted a brief TBI screening (study-trained triage nurses screened for mild TBI by asking patients two questions that were based on the CDC's clinical definition of mild TBI. Specificity of the screening tool was $95 \%$ and showed that most patients with a negative screening result were not found to have a mild TBI-related ICD-10-CM code in their record) at triage with the results not conveyed to the ED physician. ${ }^{9}$ Among those that screened positive at triage, a subsequent review of the medical record found a documented mild TBI evaluation in only $45.9 \%$ of patients and a TBI diagnosis was assigned in only $37 \%$ of cases that screened positive. These studies suggest a longstanding issue related to the underdiagnosis, and potentially underassessment, of patients in the ED for TBI. Of concern, these studies suggest many patients with a TBI will leave the ED without instruction about how to best manage their injury. A secondary concern is that these TBIs will not be included in surveillance estimates, resulting in an underestimate of the public health burden of TBI and a potentially inadequate allocation of public health resources.

Findings in this manuscript are subject to additional limitations. First, potential bias may have been introduced due to differences in methodology and medical professionals routinely documenting only positive findings in the record. The study's medical record reviewers ranged from those with expertise in the clinical assessment of TBI to professional coders, who are required to rely on the physician's documentation for diagnosis assignment. The number and types of hospitals varied across states. Sampled records were drawn from either one or multiple hospitals within a state and included critical access hospitals, acute care, trauma centres or teaching hospitals. Two of the four states sampled from $100 \%$ of the acute care hospitals in their states, while the others had less extensive coverage. Age distribution of the sampled patient population varied across states and its impact on study findings is unknown. Study years assessed varied by state: one state used the first year of data following implementation of ICD-10-CM, while the other states combined multiple study years. Therefore, readers should not compare state-specific results. Second, this study used a tiered TBI case confirmation strategy to characterise the level of certainty a TBI occurred. This strategy has not been validated and misclassification of cases is possible. However, without an objective diagnostic aid for TBI and a validated consensus clinical definition, the magnitude of potential misclassification in our study is unknown. Third, our results reflect clinical practices in hospitals within four states and findings may not be generalisable to other states or different healthcare environments. Fourth, the lack of standardised training in injury coding could influence individual hospital coding practices and potentially bias the initial sample assessed. Fifth, misclassification of sampled records is possible as accurate assessment of diagnostic coding was challenging in states where not all medical documents that were requested were received.

This study found that some sampled charts uniquely assigned S09.90 that included a documented injury event contained medium or higher evidence that a TBI occurred. Future research should seek to understand why medical records containing

\section{What is already known on this subject}

- In the International Classification of Disease, Ninth Revision, Clinical Modification (ICD-10-CM) coding era, multiple studies found that the 'unspecified head injury' codes comprised a majority of traumatic brain injury (TBI) morbidity cases in their emergency department samples. In 2016, the CDC excluded the ICD-10-CM complementary diagnosis code to 'unspecified, head injury' (S09.90) from the proposed TBI morbidity surveillance case definition.

\section{What this study adds}

Results of this multisite medical record review of sampled emergency department cases assigned S09.90 'unspecified injury of head' suggest the potential for missed TBI cases in a routinely used public health surveillance data source. Using our high and medium levels of certainty a TBI has occurred, individual state PPV ranged from $36 \%$ to $52 \%$ and represents a significant percentage of potentially missed TBI cases. 
medium-to-high levels of evidence indicative of a TBI were assigned the S09.90 code, rather than other diagnoses in the proposed TBI surveillance definition. While the primary purpose of ICD-10-CM codes is not for surveillance or research, these codes are often used to identify cases in surveillance and cohorts within research. In the ICD-10-CM era, surveillance and research efforts for TBI will be impacted as exclusion of S09.90 from CDC's TBI surveillance definition will exclude some true TBI cases, while including S09.90 will include some false-positive TBI cases.

\section{CONCLUSION}

Results of this multisite medical record review of sampled ED cases assigned S09.90 unspecified injury of head (without other TBI codes) suggest the potential for missed TBI cases when conducting surveillance using ED administrative data. Individual state PPV ranged from $36 \%$ to $52 \%$ using criteria that assigned a medium or higher level of certainty a TBI occurred. TBI diagnosis and TBI surveillance using diagnostic coding is impeded by the transitory and variable manifestations of the condition itself, as well as the lack of objective diagnostic tools.

Acknowledgements The authors thank the University of Maryland School of Medicine National Study Center for Trauma and Emergency Medical Systems and Johns Hopkins Center for Injury Research and Policy for their collaboration on this medical record review study. They thank Huong Luu, MD, MPH and GYeon Oh, MS, for their assistance with Kentucky's data analysis for this study.

Contributors The study was conceived by all authors. Data collection and analysis were performed by BAG, JJ, BH, AL, JFC, JH, SS, HL and GO. Interpretation of study findings and drafting/revision of the manuscript were performed by all authors.

Funding This work was supported in part by the Centers for Disease Control and Prevention through the following cooperative agreements: Colorado: 6NU17CE924841-03; Kentucky: 6NU17CE924846-03; Massachusetts: 6NU17CE924835-03; Maryland: 5NU17CE924831-03.

Disclaimer The findings and conclusions in this paper are those of the authors and do not necessarily represent the official position of the Centers for Disease Control and Prevention, the State of Colorado, the State of Kentucky, the State of Maryland, or the State of Massachusetts.

Competing interests None declared.

Patient and public involvement Patients and/or the public were not involved in the design, or conduct, or reporting, or dissemination plans of this research.
Patient consent for publication Not required.

Ethics approval The University of Kentucky Institutional Review Board approved the research protocol for the Kentucky data used in this study. The Maryland Department of Health, Johns Hopkins University, and University of Maryland, Baltimore Institutional Review Boards approved the research protocol used in this study.

Provenance and peer review Commissioned; externally peer reviewed.

Data availability statement Data may be obtained from a third party and are not publicly available. All data relevant to the study are included in the article or uploaded as supplementary information. N/A.

Open access This is an open access article distributed in accordance with the Creative Commons Attribution Non Commercial (CC BY-NC 4.0) license, which permits others to distribute, remix, adapt, build upon this work non-commercially, and license their derivative works on different terms, provided the original work is properly cited, appropriate credit is given, any changes made indicated, and the use is non-commercial. See: http://creativecommons.org/licenses/by-nc/4.0/.

\section{ORCID iDs}

Alexis Peterson http://orcid.org/0000-0003-3033-0177

Barbara A Gabella http://orcid.org/0000-0001-6301-116X

Svetla Slavova http://orcid.org/0000-0002-4541-6574

\section{REFERENCES}

1 Faul M, Xu L, Wald MM, et al. Traumatic brain injury in the United States: emergency department visits, hospitalizations and deaths 2002-2006. Atlanta, GA: Centers for Disease Control and Prevention, National Center for Injury Prevention and Control, 2010. https://www.cdc.gov/traumaticbraininjury/pdf/blue_book.pdf

2 Centers for Medicare \& Medicaid Services. ICD-10. Baltimore, MD: Centers for Medicare \& Medicaid Services. Available: https://www.cms.gov/Medicare/Coding/ICD10

3 Hedegaard HB, Johnson RL, Ballesteros MF. Proposed ICD-10-CM surveillance case definitions for injury hospitalizations and emergency department visits. National health statistics reports, 2016: 1-20. https://www.cdc.gov/nchs/data/nhsr/nhsr100.pdf

4 Chan V, Mann RE, Pole JD, et al. Children and youth with 'unspecified injury to the head': implications for traumatic brain injury research and surveillance. Emerg Themes Epidemiol 2015;12:9.

5 Bazarian JJ, Veazie P, Mookerjee S, et al. Accuracy of mild traumatic brain injury case ascertainment using ICD-9 codes. Acad Emerg Med 2006;13:31-8.

6 Shore AD, McCarthy ML, Serpi T, et al. Validity of administrative data for characterizing traumatic brain injury-related hospitalizations. Brain Inj 2005;19:613-21.

7 Menon DK, Schwab K, Wright DW, et al. Position statement: definition of traumatic brain injury. Arch Phys Med Rehabil 2010;91:1637-40.

8 Chen C, Peng J, Sribnick E, et al. Trend of age-adjusted rates of pediatric traumatic brain injury in U.S. emergency departments from 2006 to 2013. Int J Environ Res Public Health 2018;15:1171.

9 Koval RR, Zalesky CC, Moran TP, et al. Concussion care in the emergency department: a prospective observational brief report. Ann Emerg Med. In Press 2020;75:483-90. 